\title{
Adaptive turbo equalization for underwater acoustic communication
}

\author{
Loris Cannelli*, Geert Leus*, Henry Dol ${ }^{\dagger}$ and Paul van Walree ${ }^{\ddagger}$ \\ *Delft University of Technology, Mekelweg 4, 2628 CD Delft, The Netherlands \\ $\dagger^{\dagger}$ Acoustics and Sonar Department, TNO, The Hague, The Netherlands \\ Email: Henry.Dol@tno.nl \\ ${ }^{\ddagger}$ Maritime Systems Division, FFI, Horten, Norway \\ Email: Paul.vanWalree@ffi.no
}

\begin{abstract}
In this paper a multiband transceiver designed for underwater channels is presented. Multi-branch filtering at the receiver is used to leverage the diversity offered by a multi-scale multi-lag scenario. The multi-branch bank of filters is constructed by estimating scale and delay coefficients through an initial preamble composed by a maximum length sequence. An intelligent design of the pulse shaper at the transmitter and the receiver permits the reduction of the complexity of the equalization algorithm: the effective channel can be seen as a simple time-invariant finite impulse response filter possibly affected by a carrier frequency offset. Adaptive turbo equalization is chosen to deal with the high time-variability which characterizes many underwater channels and also to avoid the burden of estimating all parameters of the different paths. A phase locked loop and recursive least squares algorithm is implemented on each branch and for each subband which sweep the received sequence several times to refine decoding; in order to enhance the receiver performance the updating of the equalizer taps is obtained by making use of soft information provided as feedbacks from the turbo decoder. The proposed transceiver is tested on a realistic channel obtained by channel soundings performed in the Lyme Bay area, South England. Also comparisons with other existing turbo equalizer schemes are performed.
\end{abstract}

Index Terms-Multiband equalization, multi-scale multi-lag, adaptive turbo equalization, underwater communications.

\section{INTRODUCTION}

The underwater acoustic channel is one of the most challenging media for communication systems [1], [2]. One typical feature that characterizes this channel, which has to be taken into account, is the dense presence of multipath due to reflections (sea surface and/or sea bottom), refraction and scattering leading to significant delay and Doppler spreading. Furthermore the speed of sound is low $(\sim 1500 \mathrm{~m} / \mathrm{s})$ compared with the speed of light and the result is a large delay spread which in certain environments may reach the order of hundreds of milliseconds [3]. Absorption losses at high frequencies and ambient noise at low frequencies drastically limit the available bandwidth: the ratio between the signal single-sided bandwidth

This investigation has been performed in the framework of the European Defense Agency (EDA) project RACUN (Robust Acoustic Communication in Underwater Networks). The RACUN project is part of the EDA-UMS program (European Unmanned Maritime Systems for MCM and other naval applications), and is funded by the Ministries of Defense of the five participating nations: Germany, Italy, Netherlands, Norway and Sweden. and the central frequency usually exceeds the 0.20 threshold [4], so the typical narrowband assumption that holds for the majority of models for terrestrial communications is violated for this medium and an underwater communication system is almost always considered to be inherently wideband. Since the canonical model that implies the presence of frequency shifts [5] to describe the Doppler effect is not always well matched for a wideband scenario, many recent works outline the importance of a different characterization that could be better suited for a time-varying wideband framework. When the Doppler effect is present in such a communication channel the transmitted signal is considerably dilated or compressed at the receiver and can be described by means of Doppler scales [6]-[8]. This phenomenon arises particularly in underwater channels due to the low speed of sound. Surface gravity waves, sound speed fluctuations within the water column, and intentional and unintentional platform motion give rise to Doppler effects that cannot be ignored [9]. All these different elements make it difficult to characterize underwater channels: for this reason the transceiver we present in this paper is conceived to be robust and adaptable, in order to deal with this challenging medium.

Many studies are present in literature about how to mitigate the inter-symbol interference (ISI) caused by delay spread and how to design optimal equalizers. However, it is important to note that the performance of these equalization algorithms decreases when the channels are wideband and highly timevarying as for the underwater channel and new solutions have to be found [10]. In this paper we study the design of a transceiver composed by a multi-band transmitter which sends a frequency-division multiplexed (FDM) signal and by a multi-branch receiver which performs adaptive turbo equalization. A multi-branch receiver is chosen to leverage the diversity offered by the presence of different paths with different delay and scale coefficients: typical receivers used in terrestrial communications are dealing with single-scale single-lag (SSSL) or single-scale multi-lag (SSML) channels, but in the underwater framework also a multi-scale multi-lag (MSML) effect can sometimes be found.

Inspired by the results in [11], an intelligent choice of the pulse shaper permits the design of a low-complexity equalizer at the receiver: after the multi-branch matched- 
filtering and sampling, the effective channel between the transmitted and received symbols on each branch and for each subband can simply be described by a finite impulse (FIR) filter with taps depending on the physical features of the channel. For the equalization of this effective channel, adaptive turbo equalization is presented in this paper: an adaptive equalizer is chosen to design a system robust to rapidly time-varying channels. The main focus is on our choice of the type of recursive least-squares (RLS) equalizer [12], [13] which performs several sweeps on the received symbols, both forward and backward and constantly updates its taps, taking into account the information provided by a soft-input soft-output (SISO) decoder. Repeated sweeps on the received symbols and adaptive updating permit to track the changes of the channel and result in an effective countermeasure to the high variability of the channel. Moreover, the burden of 'perfect' channel estimation, which would have brought high computational costs and model uncertainties, is avoided. The thing to take into consideration is that there is a trade-off, because increasing the number of subbands will reduce the tracking capability.

For the detection of the scale and delay coefficients of the propagation paths in the channel, a bank of correlators is used; the estimation errors of the scale coefficients computed in this way leave a carrier frequency offset (CFO) that is corrected by a phase locked loop (PLL) on each branch, which is updated with the same information that is provided to the RLS by the SISO decoder. An existing receiver scheme, which uses multibranch filtering, RLS equalization and synchronization through PLLs can be found in [14], but the underlying mechanisms are different: the major difference is that the time-invariant FIR equalizer structure in [14] is not due to the pulse shape design, but due to the assumption that the channel remains constant within a message frame. Furthermore, no adaptive turbo equalization is used in [14].

Among different equalizers, RLS is chosen for its properties of fast convergence and tracking; the major drawback of this algorithm is its computational complexity, but this problem is minimized in this work by performing different RLS equalizers for the different branches [15]. If the cross-correlation between the effective channels on the branches are negligible, this scheme is optimal, but the complexity is reduced by a factor equal to the number of frequency bands.

Adaptive turbo equalization methods can be found in [16], [17], but they use only forward passes for the equalizer sweeps, which renders it difficult to deal with the variability of the channels. Moreover, unconditional hard symbol decisions in [16] and soft decisions in [17] are used for the update process, whereas conditional hard decisions are evaluated in our proposed receiver. The equalizer taps for every subband are updated in each sweep by taking into account the feedback provided by the SISO turbo decoder [18]: during each sweep, when in decision directed mode, if the symbols have been estimated and decoded reliably in the previous sweep, they are considered as reference symbols in the current sweep and the equalizers are updated, otherwise the filter coefficients remain temporarily frozen. In this way, initially only pilot symbols are reference symbols, but in the following sweeps the performance of the equalizers becomes more and more robust due to the increment of the number of reliable symbols. Note that in contrast to the equalizers, the PLLs are updated in every time step of every sweep using a decision directed approach. In [15] a multicarrier transmission scheme is presented with a similar adaptive turbo equalization structure at the receiver, but without the multi-branch filtering and with a transmitted signal that puts the same symbol sequence on each subband.

The rest of the paper is organized as follows. Section II describes the transmitter. Section III illustrates the various stages of the receiver and discusses the differences with other proposed equalizer schemes. In Section IV, results and performance characteristics are discussed for tests on realistic channels measured during sea trials; comparisons with different turbo equalizer methods are shown. Finally, Section V provides conclusions.

Notation: Uppercase boldfaced letters represent matrices, lowercase boldfaced letters represent column vectors; $(\cdot)^{*}$ indicates conjugate, $(\cdot)^{T}$ denotes nonconjugate transpose and $(\cdot)^{H}$ denotes conjugate transpose or Hermitian. $\mathbf{0}_{N}$ stands for a column vector with $N$ zeros and $\mathbf{I}_{N X N}$ indicates the $N \times N$ identity matrix.

\section{TRANSMITTER}

The transmitted signal is a multi-band signal composed of $K$ different subbands:

$$
\bar{s}(t)=\sum_{k=0}^{K-1} \sum_{n=1}^{N} s_{k, n} p(t-n T) e^{j 2 \pi f_{k} t}
$$

where

$$
f_{k}=f_{c}+\left(k-\frac{K-1}{2}\right) \Delta f
$$

are the different carrier frequencies; $f_{c}$ is the central frequency; $T$ the symbol period and $s_{k, n}$ is the $n$-th data symbol on the $k$ th subband. $\Delta f$ is the distance between the center frequencies of two adjacent subbands and, to reduce interference, it is chosen to be $\Delta f>B$, where $B$ is the band occupied by each subband. The waveform is binary phase-shift keying (BPSK) modulated and composed of $N$ symbols $s_{k, n} \in\{-1,1\}$; on each subband $N_{\mathrm{p}}$ pilot symbols and $N_{\mathrm{d}}$ data symbols are present.

Fig. 1 shows how the symbols are ordered: the initial pilot sequence on each subband is a maximum length sequence. For testing purposes the data symbols are generated from a sequence of random bits, encoded by a turbo encoder [18], and then randomly interleaved. A maximum-length sequence of BPSK symbols is sent prior to the signal, for estimation of the scale and delay coefficients of the channel. This preamble fills all the available bandwidth and it is separated from the main signal by zero-padding.

The considered pulse shape is a root raised-cosine pulse:

$$
p(t)=C \frac{\sin \left[\pi \frac{t}{T}(1-\gamma)\right]+4 \gamma \frac{t}{T} \cos \left[\pi \frac{t}{T}(1+\gamma)\right]}{\pi \frac{t}{T}\left[1-\left(4 \gamma \frac{t}{T}\right)^{2}\right]},
$$




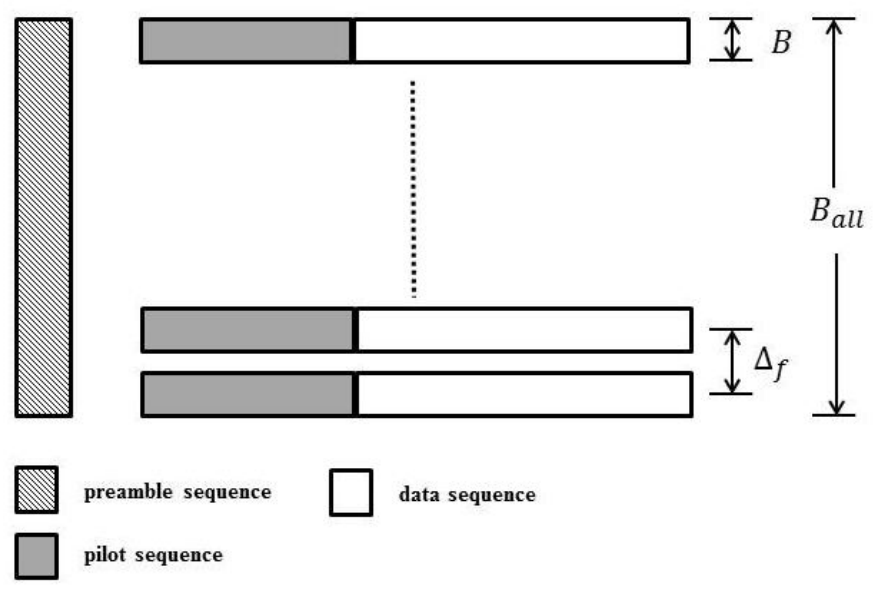

Fig. 1. Schematic representation of the structure of the transmitted message.

with $\gamma$ the rolloff factor, $C$ a normalization constant and bandwidth $B=\frac{(1+\gamma)}{T}$.

It is assumed that the transmission is in blocks, and between each block zero-padding is present to avoid inter-block interference (IBI), so it is sufficient to just focus on individual transmit blocks.

\section{RECEIVER}

The receiver is composed of different stages: initially, a bank of correlators is considered for channel parameter estimation, followed by a multi-branch bank of filters for symbol acquisition. Then one RLS equalizer and one PLL for each branch is implemented, followed by a turbo decoder that provides soft information as feedback to the equalizers in order to increment the performance and the robustness of the receiver.

\section{A. Channel estimation and symbol acquisition}

The received noiseless passband signal, according to a MSML description of the channel, can be written as:

$$
\bar{r}(t)=\sum_{q=0}^{Q-1} h_{q} \sqrt{\alpha_{q}} s\left(\alpha_{q}\left(t-\tau_{q}\right)\right),
$$

where $Q$ indicates the number of propagation paths in the channel, and where $\alpha_{q}$ and $\tau_{q}$, respectively, represent the scale and the delay coefficient of the $q$-th path.

Initially, the preamble of the received signal is correlated with a filter bank of Doppler shifted (resampled) replicas. This structure should have a good resolution in both the scale and time domain. Doppler shifts are obtained by resampling. Each correlator is associated with a scale-delay pair of coefficients, and if the output power from a certain correlator is higher than a fixed threshold, then a path with the scale-delay coefficient pair of that correlator is assumed to be present in the channel.

After this, the multi-branch bank of filters in Fig. 2 is constructed. Every branch corresponds to a different path and for each branch first the received signal is time-shifted with $-\frac{\tau_{q}}{\alpha_{q}}$, and then down-converted such that the $k$-th subband is

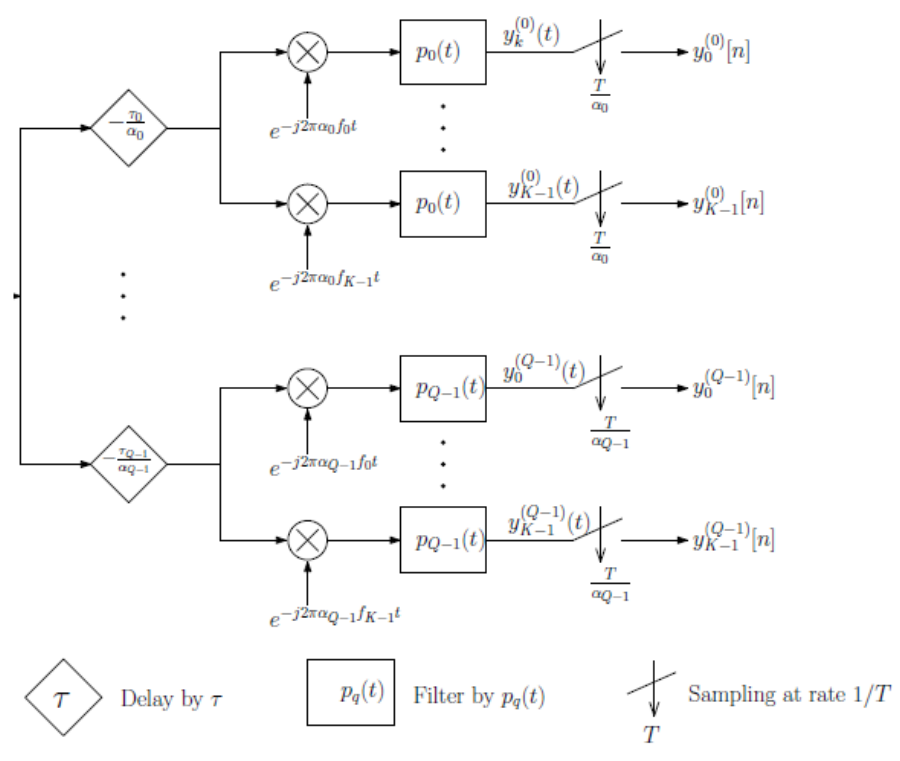

Fig. 2. Multi-branch scheme.

located at baseband, and finally the following lowpass filter is applied:

$$
p_{q}(t)=\alpha_{q}^{1 / 2} p\left(\alpha_{q} t\right) .
$$

After sampling we then obtain:

$$
y_{k}^{(q)}[n]=y_{k}^{(q)}\left(n \frac{T}{\alpha_{q}}\right) .
$$

It can be shown that, if the transmit and receive filters are designed in accord to certain properties, then the $\mathrm{I} / \mathrm{O}$ relationship for each branch and subband is that of a timeinvariant FIR filter possibly affected by a CFO [19]:

$$
y_{k}^{(q)}[n] \approx e^{j 2 \pi f_{k} n T \epsilon_{q} / \alpha_{q}} \sum_{l=0}^{L_{q}} g_{k, l}^{(q)} s_{k, n-l},
$$

where $\epsilon_{q}=\alpha_{q}-\hat{\alpha}_{q}$ and $g_{k, l}^{(q)}$ are the filter taps.

\section{B. Equalization}

As already said in the introduction, we choose a modified version of the RLS algorithm [15] to equalize the received symbols. For the $k$-th subband and the $q$-th branch we define the vector:

$$
\mathbf{y}_{k, n}^{(q)}=\left[\begin{array}{c}
y_{k}^{(q)}\left[n-\frac{\left(L_{\text {tap }}-1\right)}{2}\right] \\
y_{k}^{(q)}\left[n-\frac{\left(L_{\text {tap }}-3\right)}{2}\right] \\
\cdot \\
\cdot \\
\cdot \\
y_{k}^{(q)}\left[n+\frac{\left(L_{\text {tap }}-3\right)}{2}\right] \\
y_{k}^{(q)}\left[n+\frac{\left(L_{\text {tap }}-1\right)}{2}\right]
\end{array}\right]
$$

which is the input for the corresponding equalizer at the $n$-th time interval, where $L_{\text {tap }}$ represents the number of equalizer taps chosen. Fig. 3 shows the equalizer scheme for the $k$-th subband. As already mentioned in the introduction, several 


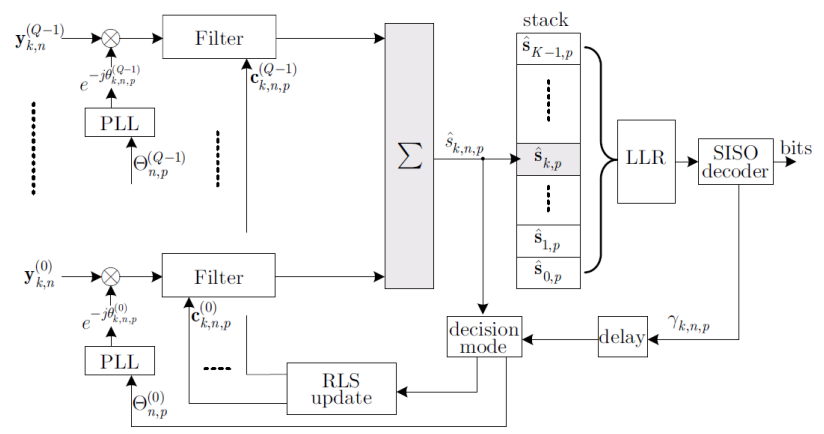

Fig. 3. Turbo adaptive equalization scheme.

equalizer sweeps are performed on the received symbols to refine the performance of the receiver. The equalizer output can be written as:

$$
\hat{s}_{k, n, p}^{(q)}=\mathbf{c}_{k, n \pm 1, p}^{(q) H} \mathbf{y}_{k, n}^{(q)} e^{-j \theta_{k, n \pm 1, p}^{(q)}},
$$

where $\hat{s}_{k, n, p}^{(q)}$ is the estimate of the $n$-th symbol over the $k$ th subband for the $q$-th branch during the $p$-th sweep; $\mathbf{c}_{k, n, p}^{(q)}$ is the vector of the corresponding equalizer taps and $\theta_{k, n, p}^{(q)}$ is the phase shift applied to the signal by the PLL for CFO correction. We assume that the sweep index $p$ is even for a forward sweep in which the symbol index $n$ increases from 0 to $N-1$ for $p=0$ and from 1 to $N-1$ in the other cases; in the backward sweeps the index $p$ is odd and the index $n$ decreases from $N-2$ to 0 . The notation in (9) summarizes this fact by selecting + in \pm for forward sweeps and - in backward sweeps. Finally, the estimate of $s_{k, n}$ in the $p$-th sweep obtained by collecting the output of all branches, is:

$$
\hat{s}_{k, n, p}=\sum_{q=0}^{Q-1} \hat{s}_{k, n, p}^{(q)} .
$$

In this work, we perform the updating of the RLS equalizer taps, and also the PLL, with the aid of the information about the reliability of the symbol estimates provided by a SISO decoder. The a priori log-likelihood ratios (LLRs) for BPSK are calculated according to:

$$
\operatorname{LLR}_{k, n, p}^{(\mathrm{in})}=\ln \left(\frac{e^{-\left(\operatorname{Re}\left[\hat{s}_{k, n, p}\right]-\mu\right)^{2} / 2 \sigma^{2}}}{e^{-\left(\operatorname{Re}\left[\hat{s}_{k, n, p}\right]+\mu\right)^{2} / 2 \sigma^{2}}}\right)=\frac{2 \mu \operatorname{Re}\left[\hat{s}_{k, n, p}\right]}{\sigma^{2}},
$$

where $\mu$ and $\sigma^{2}$ are:

$$
\mu=\frac{1}{N_{p}} \sum_{n \in \mathcal{N}_{p}} \sum_{k=0}^{K-1} \operatorname{Re}\left[\hat{s}_{k, n, p}\right] s_{k, n},
$$

and

$$
\sigma^{2}=\frac{1}{N_{p}-1} \sum_{n \in \mathcal{N}_{p}} \sum_{k=0}^{K-1}\left|\operatorname{Re}\left[\hat{s}_{k, n, p}\right]-\mu s_{k, n}\right|^{2} .
$$

(12) and (13) hold under the assumption that the symbol estimates in each sweep have a normal distribution with
TABLE I

SIMPLIFIED RLS ALGORITHM.

\begin{tabular}{ll}
\hline \hline IF $n \in \mathcal{N}_{p}$ & or $\gamma_{k, n, p-1}>\Gamma$ \\
& $\mathbf{g}_{k, n, p}^{(q)}=\frac{\mathbf{P}_{k, n \pm 1, p}^{(q)} \mathbf{x}_{k, n, p}^{(q) *}}{\lambda+\sum_{q^{\prime}}^{Q-1} \mathbf{x}_{k, n, p}^{\left(q^{\prime}\right) T} \mathbf{P}_{k, n \pm 1, p}^{q^{\prime}} \mathbf{x}_{k, n, p}^{\left(q^{\prime}\right) *},}$, \\
& $\mathbf{P}_{k, n, p}^{(q)}=\lambda^{-1}\left[\mathbf{P}_{k, n \pm 1, p}^{(q)}-\mathbf{g}_{k, n, p}^{(q)} \mathbf{x}_{k, n, p}^{(q) T} \mathbf{P}_{k, n \pm 1, p}^{(q)}\right]$, \\
& $\mathbf{c}_{k, n, p}^{(q)}=\mathbf{c}_{k, n \pm 1, p}^{(q)}+\epsilon_{k, n, p} \mathbf{g}_{k, n, p}^{(q)}$ \\
ELSE & $\mathbf{g}_{k, n, p}^{(q)}=\mathbf{g}_{k, n \pm 1, p}^{(q)}$, \\
& $\mathbf{P}_{k, n, p}^{(q)}=\mathbf{P}_{k, n \pm 1, p}^{(q)}$, \\
& $\mathbf{c}_{k, n, p}^{(q)}=\mathbf{c}_{k, n \pm 1, p}^{(q)}$ \\
END &
\end{tabular}

mean $\pm \mu$ and variance $\sigma^{2}$ on the real axis; this assumption is realistic according to the fact that it is known that the distribution of random variables at the output of a linear Wiener filter is quite close to Gaussian. We also assume that these statistics, calculated from the pilot symbols, will hold for the whole signal, which is reasonable when a sufficient number of pilot symbols is present in the signal.

With the $\operatorname{LLR}_{k, n, p}^{(\mathrm{in})}$, the SISO decoder can calculate, besides the decoded bits, the a posteriori LLR, which is used to determine the probability of the symbol estimate $\hat{s}_{k, n, p}$ to be 0 or 1 . In this way, also the reliability of the decision taken on the symbol itself is known. The probability that $\hat{s}_{k, n, p}$ is equal to 0 is:

$$
\gamma_{k, n, p}(0)=\frac{e^{\operatorname{LLR}_{k, n, p}^{\text {(out) }}}}{1+e^{\operatorname{LLR}_{k, n, p}^{\text {(out) }}}} ;
$$

and to 1 is:

$$
\gamma_{k, n, p}(1)=1-\gamma_{k, n, p}(0)
$$

The equalizer taps $\mathbf{c}_{k, n, p}^{(q)}$ are separately updated with a specific RLS scheme for each branch, according to TABLE I. In this table, $\mathbf{P}_{k, n, p}^{(q)}$ is the error covariance matrix specific for the $q$-th effective channel, $\lambda$ is the RLS forgetting factor, $\mathbf{g}_{k, n, p}^{(q)}$ is the Kalman gain vector, and

$$
\mathbf{x}_{k, n, p}^{(q)}=\mathbf{y}_{k, n}^{(q)} e^{-j \theta_{k, n \pm 1, p}^{(q)}} .
$$

This simplification of the canonical RLS algorithm [12] is valid when the different effective channels on the different branches are uncorrelated and the total error covariance matrix $\overline{\mathbf{P}}_{k, n, p}$ becomes a block diagonal matrix with the $\mathbf{P}_{k, n, p}^{(q)}$ matrices as diagonal blocks. This approximation reduces the complexity by a factor $Q$. The error signal in TABLE I is given by:

$$
\epsilon_{k, n, p}=\hat{s}_{k, n, p}-s_{k, n, p, \text { ref }}
$$

When $n \in \mathcal{N}_{\mathrm{p}}$, the algorithm is in training mode and the reference symbols are the pilots themselves:

$$
s_{k, n, p, \text { ref }}=s_{k, n}
$$


while, in decision-directed mode, $n \in \mathcal{N}_{d}$, the reference symbols are given by:

$$
s_{k, n, p, \text { ref }}= \begin{cases}-1 & \text { if } \gamma_{k, n, p-1}(1)>\Gamma, \\ +1 & \text { if } \gamma_{k, n, p-1}(0)>\Gamma, \\ \operatorname{sgn}\left\{\operatorname{Re}\left[\hat{s}_{k, n, p}\right]\right\} & \text { otherwise. }\end{cases}
$$

As can be seen in TABLE I, the equalizer taps are only updated when the estimated symbol from the previous sweep is considered reliable, otherwise the coefficients remain temporarily fixed. With consecutive sweeps, the number of reliable symbols will increase, improving the bit error ratio (BER). The filters are initialized with $\mathbf{c}_{k, 0,0}^{(q)}=\mathbf{0}_{L_{\text {tap }} \mathrm{X} 1}$ and $\mathbf{P}_{k, 0,0}^{(q)}=\mathbf{I}_{L_{\text {tap }}}$. At the boundaries of the forward and backward sweeps the following convention is used: $\mathbf{c}_{k, N, p}^{(q)}=\mathbf{c}_{k, N, p-1}^{(q)}$ and $\mathbf{P}_{k, N, p}^{(q)}=\mathbf{P}_{k, N, p-1}^{(q)}$ for odd $p$ (from forward to backward sweep), otherwise $\mathbf{c}_{k, 1, p}^{(q)}=\mathbf{c}_{k, 1, p-1}^{(q)}$ and $\mathbf{P}_{k, 1, p}^{(q)}=\mathbf{P}_{k, 1, p-1}^{(q)}$. Unlike the RLS filter, the PLL is always updated because it is less sensitive to incorrect decisions; the equations for the PLL are [14]:

$$
\begin{gathered}
\Theta_{n, p}^{(q)}=\frac{1}{K} \sum_{k=0}^{K-1} \frac{\operatorname{Im}\left\{\hat{s}_{k, n, p}^{(q)}\left(s_{k, n, p, \text { ref }}-\sum_{q^{\prime}, q^{\prime} \neq q} \hat{s}_{k, n, p}^{\left(q^{\prime}\right)}\right)^{*}\right\}}{f_{k}} \\
\eta_{n \pm 1, p}^{(q)}=\eta_{n, p}^{(q)}+(-1)^{p} \Theta_{n, p}^{(q)}, \\
\beta_{n \pm 1, p}^{(q)}=\beta_{n, p}^{(q)}+K_{1} \Theta_{n, p}^{(q)}+(-1)^{p} K_{2} \eta_{n \pm 1, p}^{(q)} \\
\theta_{k, n, p}^{(q)}=f_{k} \beta_{n, p}^{(q)}
\end{gathered}
$$

where $K_{1}$ and $K_{2}$ are the proportional and the integral phasetracking constants, respectively, and the values of $\eta_{n, p}^{(q)}$ and $\beta_{n, p}^{(q)}$ are set to zero for $n=p=0$. The convention for the behaviour of $\theta_{k, n, p}^{(q)}$ at the boundaries of the forward and backward sweeps is the same as outlined before for the filter taps. Different from [14], the term $(-1)^{p}$ is here inserted due to the presence of the backward sweep.

\section{Results}

In this section we present the results obtained after various tests performed with the proposed transceiver. The results are obtained using the channel simulation software, called MIME, developed within the RACUN consortium. This software is used to generate received signals for underwater channels according to certain specifications [20]. In particular, one of the configurations of MIME permits to mimic the impulse response of a real channel by using real measurements obtained from sea trials and to convolve this response with an input waveform; also white Gaussian noise, amongst other options, can be added with a fixed signal-to-noise ratio (SNR). The Mime replay channel simulator has been successfully validated [21], and this software is well suited to evaluate the performance of new modulation schemes in a realistic manner without new sea trials.

\section{A. Parameters}

The input waveform contains 35 identical packets, stacked in a signal of $20 \mathrm{~s}$, in order to calculate network statistics like bit error rate (BER) and package error rate (PER). Each packet consists of a 255-bit preamble, guard period, and finally the signal with pilot and data sequence. The signal is composed of $K=6$ subbands that span the frequency range from 4 $\mathrm{kHz}$ to $8 \mathrm{kHz}$; each subband occupies a bandwidth of $B \approx$ $623 \mathrm{~Hz}$ and the distance between the center frequencies of two adjacent subbands is $\Delta f=643 \mathrm{~Hz}$. On each subband, $N=75$ symbols are present, $N_{\mathrm{p}}=31$ of which constitute a maximum length sequence of pilot symbols, and $N_{\mathrm{d}}=44$ are the coded data symbols. Data symbols are generated from a sequence of 130 random bits encoded by a standard 1/2rate turbo encoder with the generator polynomial $(5,7)$, BPSK modulated, randomly interleaved and finally allocated to the subbands. The pulse shaper is designed according to (3) with a roll-off factor $\gamma=1 / 3$ and a symbol rate $1 / T=416 \mathrm{~Hz}$. With these specifications, it is possible to write the I/O relationship as in (7).

At the receiver, we design the equalizer on each branch to have $L_{\text {tap }}=3$ taps, the forgetting factor is $\lambda=0.99$ and the probability threshold is $\Gamma=0.8$. As far as the PLL is concerned, we set $K_{1}=0.02$ and $K_{2}=0.04$.

\section{B. Description of the channel}

The underwater acoustic channel used to evaluate the performance of our transceiver was measured in the Lyme Bay area, South England, in May 2011. During this sea trial, performed in the framework of the EDA-RACUN project, channel soundings were performed for different relative distances and speeds by transmitting and recording specific channel probe signals. By matched-filtering the recorded signals with the transmitted signals, impulse response evolutions are derived, which enable replay of the measured channel in the laboratory. For the particular channel, considered in this paper, the transmitter was deployed at about $5 \mathrm{~m}$ depth below a drifting buoy, while the receiver was at about $400 \mathrm{~m}$ (relative) distance, moving away with a (relative) speed of about $3 \mathrm{~m} / \mathrm{s}$ from the transmitter. The receiver was an array of hydrophones, towed horizontally at a depth of $15-20 \mathrm{~m}$ by a surface ship. A single hydrophone recording is selected for the present analysis. The sea floor was quite flat and sandy and the water depth was about $45 \mathrm{~m}$.

Fig. 4 shows in panel (a) the power delay profile of the channel, characterized by several strong arrivals even after some tens of milliseconds; the Doppler spectrum present in panel (b) of the same figure is quite wide due to the receiver motion. The width of the main lobe of the Doppler spectrum suggests the presence of a multi-scale effect: the lines in Fig. 5 which denote the different arrivals have a slight curvature highlighting a time-varying time delay caused by the Doppler effect.

However, the performance comparison in TABLE II shows that the multi-scale effect is not very significant. In TABLE II the channel to which we are referring is channel 1, but results for other different channels are presented too. The channel 


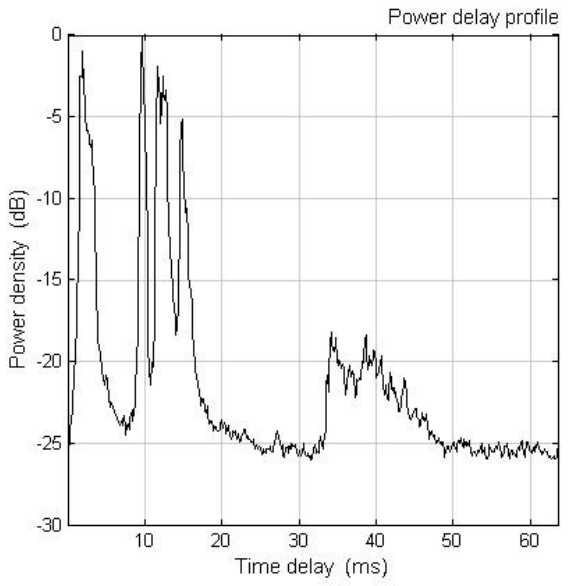

(a)

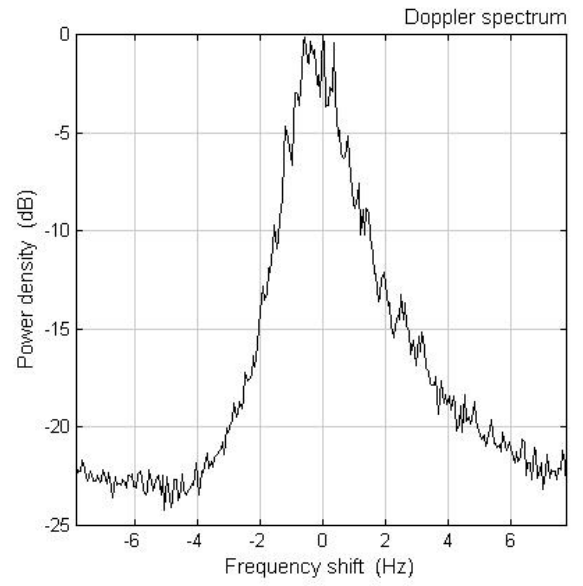

(b)

Fig. 4. Power delay profile in panel (a) and Doppler spectrum in panel (b). The Doppler spectrum is shown after complete removal of the mean Doppler shift.

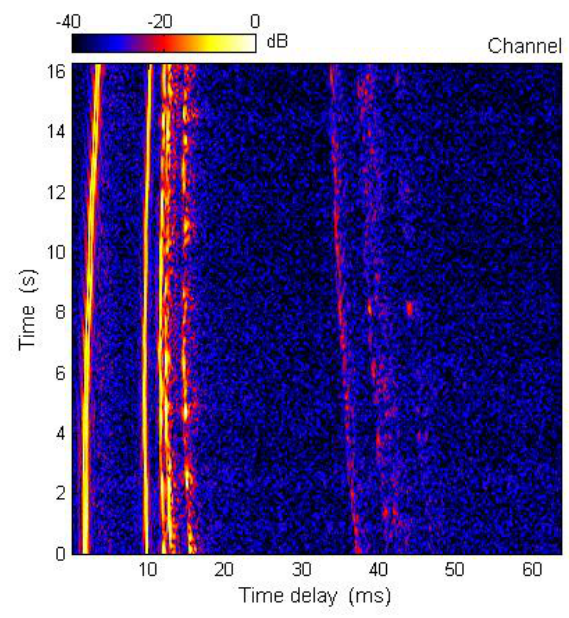

Fig. 5. Time-delay representation of the impulse response of the channel.

soundings $2-5$ come from practical measurements collected in the same sea trial described above. The performances in TABLE II are obtained with different setups of the receiver as described in this paper: the column 'single-scale singlelag (SSSL)' refers to a receiver that filters the received signal only by using the scale and delay coefficients of the path with the strongest gain; the column 'single-scale multi-lag (SSML)' shows the results of a receiver that, differently from the previous one, also takes into consideration delay coefficients of other significant paths in addition to the strongest one if they are present; finally, the last column contains the results obtained with a multi-branch receiver that also takes into consideration all the significant scale coefficients. TABLE II shows that for this channel the multi-lag effect has to be taken into consideration, but the multi-scale effect does not influence the performance. The packet error rate (PER) is calculated by considering a packet erroneous if at least one bit is not correctly received; the SNR is fixed to $5 \mathrm{~dB}$.

\section{Performance comparisons}

Fig. 6 shows the gain in performance obtained after consecutive equalizer sweeps. After each sweep the number of reliable symbols increases thanks to the information provided by the SISO decoder. So after some iterations, the equalizer can take advantage of more reference symbols than only the pilot ones, and in this way the robustness of the algorithm is better than of those algorithms that always update the equalizer coefficients. In Fig. 6 it is possible to see that there is only a small gain in performance from the 5th sweep to the 10th sweep;on the other hand, the computation time is seen to increase about linearly with the number of the sweeps. Magenta curve is showed as an asymptotic reference and it is obtained with the equalizer that knows all the transmitted symbols and so operates in training mode throughout the entire signal. Fig. 7 shows the equalized symbols, without the initial training, in the complex plane. The BPSK data in panel (a) are grouped in only one cloud quit close to zero: symbols further from zero will become reference symbols in the successive sweeps. In the following sweeps, such as the 10th sweep in panel (b), it is possible to notice that the symbols form two groups biased towards -1 or +1 . In [16], the RLS coefficients are constantly updated with hard decisions taken from the equalized symbols exiting the turbo decoder. The reliability of the decisions is not considered and unconditional hard decisions are used; so when the algorithm is in the decision directed mode we can modify (19) for [16] to:

$$
s_{k, n, p, \text { ref }}= \begin{cases}-1 & \text { if } \gamma_{k, n, p-1}(1)>0.5 \\ +1 & \text { if } \gamma_{k, n, p-1}(0)>0.5\end{cases}
$$

In this case we put $\Gamma=0.5$, which is equivalent to constantly updating the equalizers using the decisions from the previous sweep. Also in [17], turbo equalization is used: in this work the equalization algorithm is constantly updated too, but soft decisions are used to perform the iterative computation of the 
TABLE II

COMPARISON AMONG DIFFERENT RECEIVERS IN ORDER TO CHARACTERIZE THE FEATURES OF THE CHANNELS. THE RELATIVE SPEED BETWEEN TRANSMITTER AND RECEIVER IS ABOUT 3-4 M/s.

\begin{tabular}{|c|c|c|c|c|c|}
\hline & & \multicolumn{4}{|c|}{ SSSL } \\
\hline Channel number & TX-RX distance & BER $_{\text {1st sweep }}$ & BER 10th sweep $_{\text {1 }}$ & PER $_{\text {1st sweep }}$ & PER $_{10 \text { th sweep }}$ \\
\hline 1 & $400 \mathrm{~m}$ & 0.27 & 0.07 & 0.97 & 0.72 \\
\hline 2 & $550 \mathrm{~m}$ & 0.31 & 0.12 & 0.94 & 0.67 \\
\hline 3 & $750 \mathrm{~m}$ & 0.29 & 0.08 & 1 & 0.64 \\
\hline 4 & $900 \mathrm{~m}$ & 0.19 & 0.04 & 0.83 & 0.44 \\
\hline \multirow[t]{3}{*}{5} & $1500 \mathrm{~m}$ & 0.18 & 0.04 & 0.81 & 0.64 \\
\hline & & \multicolumn{4}{|c|}{ SSML } \\
\hline & & BER $_{\text {1st sweep }}$ & BER $_{10 \text { th sweep }}$ & PER $_{\text {1st sweep }}$ & PER $_{10 \text { th sweep }}$ \\
\hline 1 & $400 \mathrm{~m}$ & 0.07 & 0 & 0.75 & 0.11 \\
\hline 2 & $550 \mathrm{~m}$ & 0.12 & 0.02 & 0.83 & 0.22 \\
\hline 3 & $750 \mathrm{~m}$ & 0.6 & 0 & 0.78 & 0.06 \\
\hline 4 & $900 \mathrm{~m}$ & 0.09 & 0.01 & 0.83 & 0.22 \\
\hline \multirow[t]{3}{*}{5} & $1500 \mathrm{~m}$ & 0.06 & 0.01 & 0.78 & 0.25 \\
\hline & & \multicolumn{4}{|c|}{ MSML } \\
\hline & & BER $_{\text {1st sweep }}$ & BER $_{10 \text { th sweep }}$ & PER $_{\text {1st sweep }}$ & PER $_{10 \text { th sweep }}$ \\
\hline 1 & $400 \mathrm{~m}$ & 0.08 & 0 & 0.78 & 0.11 \\
\hline 2 & $550 \mathrm{~m}$ & 0.12 & 0.02 & 0.81 & 0.23 \\
\hline 3 & $750 \mathrm{~m}$ & 0.6 & 0 & 0.78 & 0.06 \\
\hline 4 & $900 \mathrm{~m}$ & 0.09 & 0 & 0.81 & 0.19 \\
\hline 5 & $1500 \mathrm{~m}$ & 0.06 & 0.01 & 0.75 & 0.25 \\
\hline
\end{tabular}

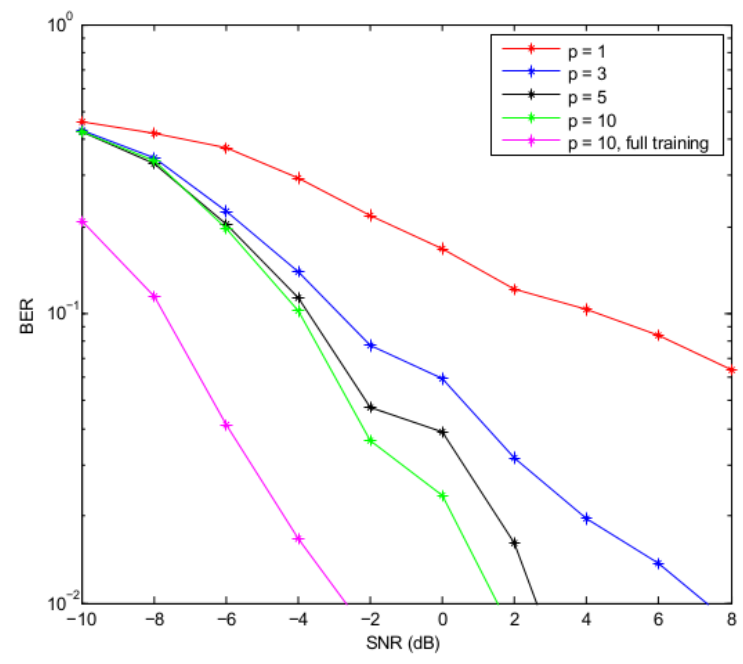

Fig. 6. BER curves plotted for different sweeps (1st, 3rd, 5th and 10th) of the iterative RLS equalizer. Magenta curve is plotted as a reference and it is the BER curve for the 10th sweep when the equalizer is in full training mode.

new coefficient. In [17], the error signal (17), which drives the evolution of the equalizer coefficients, is calculated directly from the soft information about the decoded symbol in the previous sweep; we can imitate this idea modifying (19) as:

$$
s_{k, n, p, \text { ref }}=\left\{\begin{array}{l}
\gamma_{k, n, p-1}(0)-\gamma_{k, n, p-1}(1) \\
\text { if } \gamma_{k, n, p-1}(0)>0.5 \vee \gamma_{k, n, p-1}(1)>0.5 .
\end{array}\right.
$$

According to this equation, the reference symbol is always chosen to be a real number between $[-1 ; 1]$ on the basis of the soft information provided by $\gamma_{k, n, p-1}(1)$ and $\gamma_{k, n, p-1}(0)$. Also in this case, $\Gamma$ is chosen to be equal to 0.5 to permit the continuous updating of the algorithm. In both [16], [17], backward sweeps are not present: the equalizer runs only with forward passes on the received symbols. Fig. 8 shows the results of the comparison, for the same equalizer sweep ( $p=4$ is chosen), between the methods introduced above. Curve D is obtained in full training mode i.e., as for the magenta curve in Fig. 6, with the equalizer that knows all the transmitted symbols. This curve is plotted as an asymptotic reference. Curve B and curve C are obtained according to (24) and (25), respectively. A comparison of these two curves with curve A demonstrates the advantages of using our method with respect to the other schemes in which the update of the equalizer coefficients is always performed, without considering the reliability of the symbol estimates which is used to compute the error signal in (17). We noticed that the absence of backward sweeps has a remarkable contribution to the worsening of the 


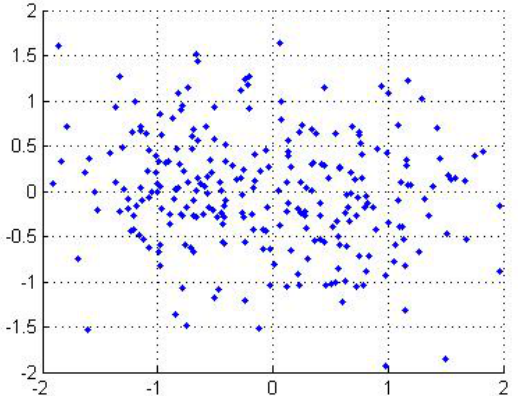

(a)

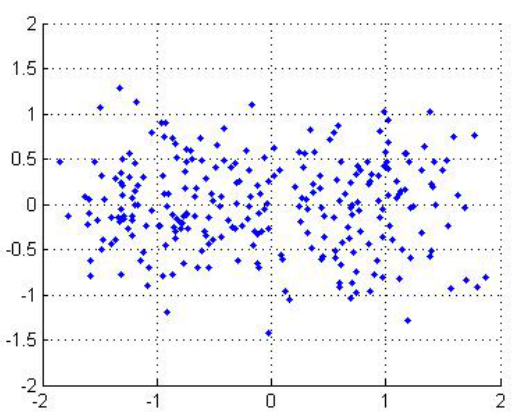

(b)

Fig. 7. Constellation of equalized BPSK symbols in the complex plane, with SNR $=8 \mathrm{~dB}$. (a) 1st equalizer sweep ( $\mathrm{p}=1$ ). (b) 10th equalizer sweep ( $\mathrm{p}=10$ ).

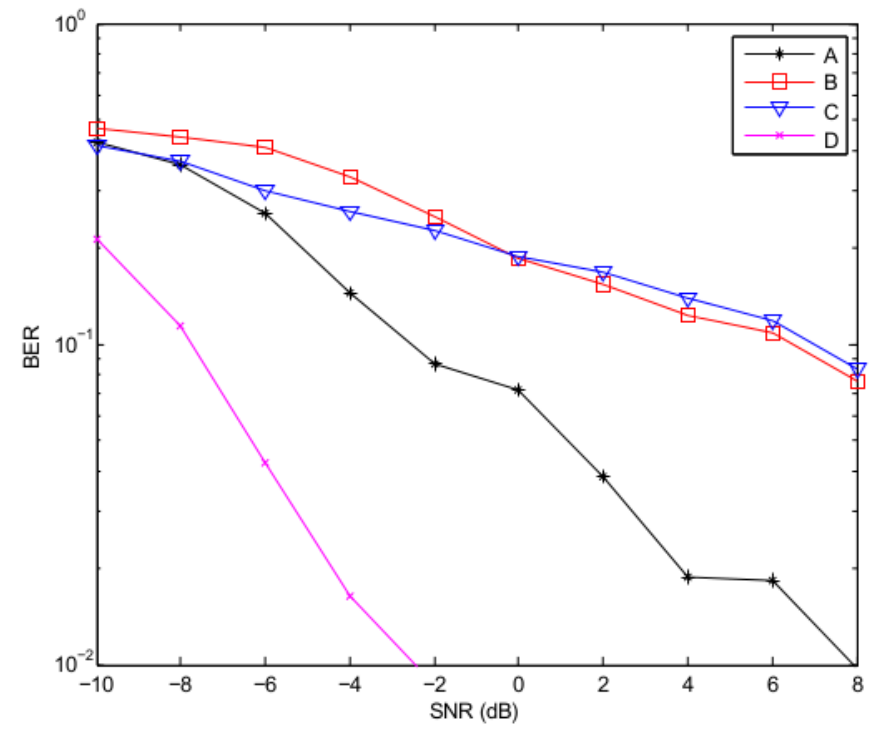

Fig. 8. (A) Proposed receiver. $(\mathrm{p}=4)(\mathrm{B})$ Receiver which uses unconditional hard decisions and forward sweeps only. ( $\mathrm{p}=4)(\mathrm{C})$ Receiver with uses unconditional soft decisions and forward sweeps only. $(p=4)(D)$ Equalizer in full training mode. $(p=4)$

performance of curves B and C.

\section{CONClusion}

A particular transceiver for challenging channels such as the underwater acoustic channels is presented in this paper. A multi-band transmitter and multi-branch receiver are developed to leverage the diversity offered by the time-varying framework while an intelligent design of both transmitter and receiver filters are designed reduces the complexity of the channel estimation: the discrete signal at each branch can be characterized by a simple time-invariant FIR system and this fact allows us to implement an adaptive RLS algorithm in order to perform equalization. The key points presented in this paper concern modifications to the canonical RLS scheme. Initially, in order to reduce complexity costs, the canonical RLS algorithm is simplified after some considerations about correlations which are often valid in underwater channels. Then turbo equalization is applied by updating the equalizer coefficients taking the soft information provided by a SISO decoder into account. The attention to the reliability of the decoded symbols and the presence of both forward and backward equalizer sweeps make the receiver robust to challenging channels, while other equalizer methods are less efficient due to the fact that for example the equalizer runs according to unconditional decisions taken on the decoded symbols. Simulations evaluated for a realistic channel confirm the robustness of our architecture.

\section{REFERENCES}

[1] D. Brady and J. C. Preisig, Wireless communications: a signal processing perspective. Prentice-Hall, March 1998, ch. 8, p. 330.

[2] J. Preisig, "Acoustic Propagation Consideration for Underwater Acoustic Communications Network Development" in Proc. WUWNet06, Sept. 2006.

[3] D. Kilfoyle and A.B. Baggeroer, The State of the Art in Underwater Acoustic Telemetry, IEEE J. Oceanic Engineering, 25(1) pp. 4-27, (2000).

[4] Federal Communications Commission, "Revision of part 15 of the commission's rules regarding ultra-wideband transmissions systems, First 
report and order", ET Docket 98-153, FCC 02-48, pp. 1-118, Feb. 14, 2002.

[5] P. Bello, "Characterization of randomly time-variant linear channels", IEEE Transactions on Communications Systems, 11(4):360-393, 1963.

[6] Y. Jiang and A. Papandreou-Suppappola, "Discrete time-scale characterization of wideband time-varying systems", IEEE Transactions on Signal Processing, 54(4),:1364-1375, 2006.

[7] A. R. Margetts, P. Schniter and A. Swami. "Joint scale-lag diversity in wideband mobile direct sequence spread spectrum systems", IEEE Transcations on Wirelesse Communications, 6(12):4308-4319, 2007.

[8] S. Rickard, R. Balan, V. Poor and S. Verdu, "Canonical time-frequency, time-scale, and frequency-scale representations of time-varying channels", Journal of Communication and Information Systems, 5(5):1-30, 2005.

[9] M. Stojanovic and J. C. Preisig, "Underwater acoustic communication channels: propagation models and statistical characterization", IEEE Commun. Mag., vol. 47, no. 1, pp. 84-89, January2009.

[10] J. W. Choi, T. Riedl, K. Kim, A. Singer, and J. Preisig, "Adaptive linear turbo equalization over doubly selective channels", Oceanic Engineering, IEEE Journal of, vol. 36, no. 4, pp. 473 489, oct. 2011.

[11] T. Xu, G. Leus and U. Mitra, ”Orthogonal wavelet division multiplexing for wideband time-varying channels". In Proc. IEEE Int. Conf. Acoust., Speech, Signal Process. (ICASSP), volume 5, pages 3556-3559, Prague, Czech, 2011.

[12] J. G. Proakis, Digital Communications, 4th ed.New York:McGraw-Hill, 2001.

[13] S. Haykin, Adaptive Filter Theory, Englewood Cliffs, NJ; Prentice Hall, 1986.

[14] M. Stojanovic, J. Catipovic and J. G. Proakis, "Adaptive multichannel combining and equalization for underwater acoustic communications", Journal of the Acoustical Society of America, 94(3):1621-1631, 1993.

[15] P. A. van Walree and G. Leus, "Robust underwater telemetry with adaptive turbo multiband equalization", IEEE Journal of Oceanic Engineering, 34(4): 645-655, 2009.

[16] S. Roy, T. M. Duman, V. McDonald and J. G. Proakis, "High-rate communication for underwater acoustic channels using multiple transmitters and space-time coding: Receiver structures and experimental results", IEEE J. Ocean. Eng., vol. 32, no. 3, pp. 663-688, Jul. 2007.

[17] C. Laot, A. Glavieux and J. Labat, "Turbo equalization: adaptive equalization and channel decoding jointly optimized", IEEE J. Sel. Areas Commun., vol. 19, no. 9, pp. 1744-1752, Sep. 2001.

[18] C. Heegard and S. B. Wicker, Turbo Coding,New York:Springer-Verlag, 1998.

[19] T. Xu, "Wireless Transceiver Design for High Velocity Scenarios", Chapter 6.

[20] P. A. van Walree, T. Jenserud and M. Smedsrud, "A discrete-time channel simulator driven by measured scattering functions", IEEE J. Sel. Areas Commun., vol. 26, no. 9, pp. 1628-1637, Dec. 2008.

[21] R. Otnes, P. A. van Walree and T. Jenserud, "Validation of direct and stochastic replay using the Mime acoustic communication channel simulator", in UComms Conference, pp. 12-14, Sep. 2012. 